\title{
The stress statistics of the first pop-in or discrete plastic event in crystal plasticity
}

\author{
P. M. Derlet ${ }^{1, a)}$ and R. Maaß ${ }^{2}$ \\ ${ }^{1}$ Condensed Matter Theory Group, Paul Scherrer Institute, CH-5232 Villigen PSI, Switzerland \\ ${ }^{2}$ Department of Materials Science and Engineering, University of Illinois at Urbana-Champaign, \\ 1304 West Green Street, Urbana, Illinois 61801, USA
}

(Received 19 July 2016; accepted 24 November 2016; published online 8 December 2016)

\begin{abstract}
The stress at which the first discrete plastic event occurs is investigated using extreme value statistics. It is found that the average of this critical stress is inversely related to the deforming volume, via an exponentially truncated power-law. This is demonstrated for the first pop-in event observed in experimental nano-indentation data as a function of the indenter volume, and for the first discrete plastic event seen in a dislocation dynamics simulation. When the underlying master distribution of critical stresses is assumed to be a power-law, it becomes possible to extract the density of discrete plastic events available to the crystal, and to understand the exponential truncation as a break-down of the asymptotic Weibull limit. Published by AIP Publishing.

[http://dx.doi.org/10.1063/1.4971871]
\end{abstract}

\section{INTRODUCTION}

Intermittent plastic activity is of contemporary interest since the phenomenon exhibits some degree of criticality and thus the universal physics of avalanche phenomenon. ${ }^{1}$ First demonstrated via acoustic emission during creep experiments on ice, ${ }^{2,3}$ the obtained histograms of energy release were found to exhibit a power law form over a wide range of energies. In the work by Miguel et al. 2, two dimensional dislocation dynamics simulations gave similar results. Subsequent dislocation dynamics simulation ${ }^{4-7}$ and theory work $^{8-10}$ have repeatedly shown this same behaviour in terms of the statistics of the plastic strain magnitudes. Another experimental approach to measuring intermittent plasticity is via the "smaller is stronger" paradigm first discussed by Uchic and co-workers. ${ }^{11}$ Here, due to the micron sized samples, a nominal flat-punch nano-indenter could resolve individual plastic events to demonstrate the intermittent plasticity and some aspects of scale free behaviour. ${ }^{12-14}$

The above works have mainly concentrated on the statistics associated with the discrete plastic event, involving either energy release, plastic strain magnitude, or the avalanche velocity, ${ }^{6,15}$ but not that of the critical stress at which the event occurs. This focus has partly arisen from the view that the plastic strain event is the material "response" to the "stimulus" of an applied stress, and that in near criticality, this material response is only weakly correlated with the stimulus. Despite this, the stress variable has played an important role in recent works investigating the theme of to what degree a material is in a state of criticality. When the global yield of a material is viewed as a static depinning transition, ${ }^{9,10,14}$ criticality is only achieved at the depinning stress. Below this stress, the statistics associated with the plastic strain event is truncated from a pure power law distribution by scaling functions depending on how far away the

a)Peter.Derlet@psi.ch applied stress is from the critical stress. The alternative view of yield as a dynamic unjamming event ${ }^{16,17}$ has the material in a critical state at all applied stresses. ${ }^{7}$ Here, the dislocation dynamics simulations show a stress and system size dependent truncation of the plastic strain statistics which does not diverge at a certain external stress.

From an engineering perspective, the stresses at which plasticity occurs are of crucial importance. If the onset of plasticity is associated with the global failure of a material, one very useful approach to understand the statistics of the failure stress is via the weakest link principle associated with extreme value theory-the so-called Weibull approach. ${ }^{18,19}$ Here, a realization of a particular material is given by a sequence of $M$ critical stresses, the smallest (weakest) of which will correspond to the stress at which the material globally fails. For a flaw-based failure scenario, these critical stresses correspond to and characterize the $M$ regions within the material containing the flaws. Mathematically, such a characterization of the material is given by a positive valued master probability density function (PDF) of critical stresses and $M=\rho V$ where $\rho$ is the flaw density. Sampling this master distribution $M$ times, the statistics of the smallest value is found to be well described by the Weibull distribution. ${ }^{20}$ Indeed, the Fisher-Tippett-Gnedenko (FTG) theorem ${ }^{21,22}$ states that for a very broad class of positive valued probability distributions and for sufficiently large values of $M$, the statistics of the minimum (extreme) value is well described by a Weibull distribution with the corresponding scale and shape parameters that do depend on the probability distribution and $M$.

For strongly heterogeneous ceramics and brittle metals, in which global failure is known to originate from a single local flaw, Weibull statistics can well describe the fluctuations in the corresponding failure stress. However, for a general crystalline metal, yield is an emergent phenomenon associated with the collective behaviour of the underlying 
dislocation network, and extreme value statistics or the weakest link principle is not expected to be so useful.

It has however long been recognized that the onset of permanent deformation is very dependent on the instrumental resolution of the deformation apparatus and that the transition to global yield is preceded by a plasticity that Chalmers first termed as the micro-plasticity. ${ }^{23}$ Indeed, many years ago Tinder and co-workers, ${ }^{24,25}$ using torsion with an incredible strain resolution of $\sim 10^{-8}$, showed such plasticity could occur in the $\mathrm{Cu}$ and $\mathrm{Zn}$ crystals well below their known yield stresses via discrete and intermittent plastic events. Between such plastic events, perfectly elastic regions of deformation were observed with corresponding moduli comparable to that determined by ultrasound techniques. This latter aspect demonstrated the high quality of these early torsion experiments.

Thus, with sufficient strain resolution, the transition to bulk yield is a gradual but discrete process mediated by intermittent plasticity - a viewpoint that is quite consistent with the modern work of stochastic plasticity, which indicates that at low enough stresses, the strain displacement distributions associated with the initial plastic events are truncated power-laws resulting in a local, less collective plasticity. ${ }^{7,9,10,14}$ This regime of plasticity should therefore be amenable to the extreme value statistics framework, where the material admits a density of uncorrelated regions defined by critical stresses at which local plasticity can be initiated. The corresponding master distribution (along with $M=\rho V$ ) would therefore characterize the underlying microstructure prior to loading in terms of these critical stresses. This approach has been taken in Refs. 26 and 27 to reveal a size effect in the onset of plasticity for uniformly loaded samples. Here, the larger the deforming volume is, the greater the $M$ is, and therefore, the lower the initial critical stresses are. For the case of bulk plasticity and its associated stress-strain curve, Derlet and Maa $\beta^{26}$ found that such a size effect is negated by the opposing size effect in plastic strain where all local plastic strain magnitude scales are inversely related to the sample volume, a result emanating from Eshelby's classic plastic-inclusion work. ${ }^{28}$

The present work applies the extreme value statistics approach to the critical stress associated with the first plastic event of a generic intermittent plastic deformation sequence. Sec. II develops the needed procedure to predict the Weibull distribution describing the statistics of the first critical stress for sufficiently large system sizes and discusses how deviations away from the asymptotic Weibull form can be used to obtain an estimate of the density of available plastic events. Sec. III applies the developed framework to rationalize recently published pop-in stress statistics of nano-indentation data as a function of the indenter size, ${ }^{29,30}$ as well as the first critical stresses obtained from dislocation dynamics simulations in the presence of a fixed internal stress field. ${ }^{5}$ Sec. IV concludes with a discussion of the consequences of these findings, and what other experimental and simulation data the developed procedure could be applied to.

\section{THE STATISTICS OF THE FIRST CRITICAL STRESS}

As discussed in Sec. I, one approach to quantify the statistics of the first critical stress is to assume that the material can admit $M$ plastic events and that the corresponding critical stresses are derived from an underlying master PDF, $P[\sigma]$, which characterizes the initial plastic response of the material. The stress statistics of intermittent plasticity is then embodied in the order statistics ${ }^{31}$ of this sequence of $M$ critical stresses. In practice, this is done by sampling $P[\sigma], M$ times, and arranging the resulting stresses in ascending order: $\left\{\sigma_{1}, \ldots, \sigma_{M}\right\}$, where $\sigma_{1}$ is the first critical stress and the focus of the present work.

For sufficiently large $M$ (sample volume), the fluctuations of $\sigma_{1}$ are described by a Weibull distribution defined by a scale parameter and a shape parameter. Whilst the Weibull form is independent of $P[\sigma]$, the actual values of the scale and shape parameters do depend on $P[\sigma]$. In particular, the scale parameter, $\sigma_{1}^{*}$, may be determined from the definition $26,31,32$

$$
\frac{1}{M} \doteq \int_{0}^{\sigma_{1}^{*}} d \sigma P[\sigma]=P_{<}\left[\sigma_{1}^{*}\right]
$$

Here, $P_{<}[\sigma]$ is the cumulative distribution function (CDF) of $P[\sigma]$. The identification of $\sigma_{1}^{*}$ with the scale parameter of the Weibull distribution is only valid in the asymptotic limit of large $M$ and therefore small $\sigma_{1}^{*}$. In this regime, it is assumed that the master distribution has the power-law form

$$
P[\sigma] \sim \sigma^{\alpha},
$$

giving, via Eq. (1),

$$
\sigma_{1}^{*} \sim\left(\frac{1}{M}\right)^{\gamma}
$$

where $\gamma=1 /(1+\alpha)$. A power-law form is motivated by the fact that many interacting systems are known to exhibit such behaviour-see, for example, the recent review on marginal stability where the master distribution is referred to as the pseudo-gap function. ${ }^{33}$

For asymptotically large $M$, Appendix A demonstrates that $1 / \gamma$ is the Weibull shape parameter, and $\sigma_{1}^{*}$ is the Weibull scale parameter. Thus, via the scaling in Eq. (3), the Weibull statistics of the first critical stress is fully defined. One immediate result is the average first critical stress, $\left\langle\sigma_{1}\right\rangle$, is linearly related to $\sigma_{1}^{*}$ via

$$
\left\langle\sigma_{1}\right\rangle[x] \simeq \Gamma[1+\gamma] \sigma_{1}^{*}[x],
$$

where $x \doteq 1 / M$ and $\Gamma[\cdot]$ is the gamma function. This provides a direct method to determine $\sigma_{1}^{*}$ via the average value of the first critical stress obtained from either experiment or simulation. Indeed, via the assumption $M=\rho V$, Eq. (3) can be tested through a study of the average first critical stress versus the plastic volume.

It is emphasized that the approach entailed in Eqs. (1)-(4) is valid only for asymptotically large $M$. One goal of the present work is to investigate how well the above holds for finite values of $M$. In fact, it is demonstrated in Sec. III that the average first critical stress seen in experiment and simulation is well described by 


$$
\left\langle\sigma_{1}\right\rangle[x]=\sigma_{0} e^{-l x} x^{\gamma},
$$

for $0 \leq x<x_{\mathrm{c}}=\gamma / l$. Here, $\sigma_{0}, l$, and $\gamma$ are parameters to be determined. Using $M=\rho V$, this may be rewritten as

$$
\left\langle\sigma_{1}\right\rangle[V]=\frac{\sigma_{0}}{\rho^{\gamma}} e^{-l /(\rho V)}\left(\frac{1}{V}\right)^{\gamma}=\bar{\sigma}_{0} e^{-\bar{l} / V}\left(\frac{1}{V}\right)^{\gamma} .
$$

Eq. (6) is fitted directly to $\left\langle\sigma_{1}\right\rangle$ versus $V$ with the parameters being $\bar{\sigma}_{0}, \bar{l}$, and $\gamma$. For sufficiently large $M, \gamma$ and $\sigma_{1}^{*}$ (via Eq. (4)) can be identified as the Weibull shape and scale parameters, respectively.

The above gives $\gamma$ and therefore $\alpha$, which is the exponent for the master distribution of critical stresses and therefore a material parameter characterizing the initial micro-structure. The above expressions are valid for a homogeneous external stress field. For a nano-indentation geometry, a generalization to an inhomogeneous external stress field is needed. This is done in Appendix B. Concerning the validity of the present formalism, $M$ is an integer which must be greater than one. Thus, for sufficiently small volumes (which might not contain a plastic event), $M$ will be mainly zero with $\langle M\rangle<0$. In this regime of small enough sample volume, the present formalism can no longer be applied and reflects a possible change of mechanism - a limit that is encountered experimentally in the present work, when sufficiently small volumes are probed with nano-indentation.

Whilst the procedure concerned with Eq. (6) can yield both the scale and shape parameter, no insight into the material parameter $\rho$, and therefore $M$, can be gained. Moreover, since $\bar{l}=l / \rho$ and $\bar{\sigma}_{0}=\sigma_{0} / \rho^{\gamma}$, the fundamental parameters $l$ and $\sigma_{0}$ are also not determined. Assuming the master distribution is a power-law for all values of relevant critical stresses, Appendix C develops an exact expression for the average first critical stress as a function of $M$, which closely follows the empirical form of Eq. (5) when $M>10$. In this developed expression, which essentially characterizes the breakdown of the asymptotic Weibull limit, the parameter $l$ is now a function of $\gamma$ (see Eq. (C8) and Fig. 5(a) in Appendix C), allowing $\rho$ and $\sigma_{0}$ to be now directly estimated. In addition, the analysis of Appendix $\mathrm{C}$ demonstrates that when $M>50$, the asymptotic Weibull limit is well followed.

\section{APPLICATION TO EXPERIMENT AND SIMULATION}

\section{A. First pop-in stress from experimental nano-indentation data}

Nano-indentation provides a reliable and accurate probe into the plastic properties of a material region directly below the indenter tip. The initial elastic response is well described by Herzian contact theory, ${ }^{34}$ and the non-negligible plasticity generally manifests itself as an abrupt deviation from Herzian behaviour. The critical stress at which this discrete plastic event occurs is referred to as a pop-in stress. In a recent series of papers, Pharr and co-workers, ${ }^{29,30}$ measured such pop-in stresses of a (100) Mo single crystal for different indenter radii ranging from $700 \mu \mathrm{m}$ down to $0.56 \mu \mathrm{m}$. The work is distinguished by the very large number of nano-indentations per intender size, and the range of indenter sizes. They found that with decreasing indenter radius, the critical pop-in stress increased in a systematic way. In Sudharshan Phani et al., ${ }^{30}$ this trend was rationalized via a stochastic deformation model involving two microscopic deformation mechanisms - the general activation of preexisting dislocations and the nucleation of dislocations in dislocation free environments for the smallest indenter radii.

The present work considers such pop-in stresses as the first critical stress of the formalism developed in Sec. II. In doing so, it is implicitly assumed that the statistics of the first plastic event arises directly from the physics of the underlying dislocation network and not, for example, from a distribution of surface imperfections-unknowns that are always present in such an experiment.

For each indenter radius, $r$, the critical stresses are taken directly from figure 10 of the study by Sudharshan Phani et al., ${ }^{30}$ averaged and plotted in Fig. 1(a) as a function of the indenter radius. Also included in Fig. 1(a) is a fit to Eq. (6) assuming $V=r^{3}$. Comparison of this fit to the experimental nanoindentation data is good apart from the largest and two smallest indenter radii. The obtained parameters are
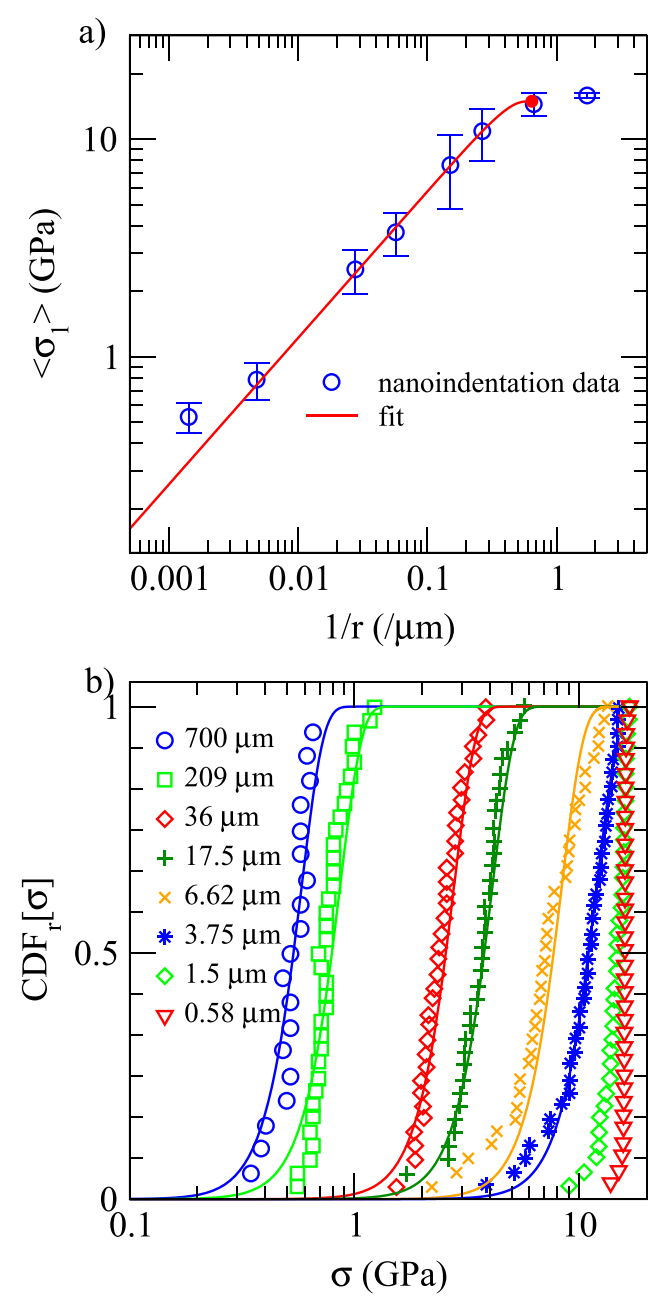

FIG. 1. (a) Logarithmic plot of measured pop-in stress as a function of the indentation radius, and an associated fit of Eq. (6). (b) Plot of the experimental indentation cumulative distribution function (CDF) data for each indenter radius with the corresponding predicted Weibull CDF. Experimental indentation data taken from figure 10 of the work by Sudharshan Phani et al. ${ }^{30}$ 
$\bar{\sigma}_{0}=27.123 \pm 0.979 \mathrm{GPa}(\mu \mathrm{m})^{-\gamma}, \bar{l}=1.189 \pm 0.105 \mu \mathrm{m}$, and $\gamma=0.225 \pm 0.006$.

By performing such a fit, it is asserted that $M=\rho V$ $=\rho r^{3}$ and that the relevant plastic volume depends only on the length scale associated with the indenter radius. In fact, the relevant plastic volume scales with the cube of the contact area radius $a$ and not the indenter radius $r$. The two length scales are however related through the indentation depth, $h$, via $a=\sqrt{r h}$. Since the stress within this plastic volume scales as $\sqrt{h / r}=a / r$ (see Refs. 34-36), a higher stress corresponds to a larger indentation depth, therefore a larger contact area and thus finally a larger plastic volume. This additional scaling must be taken into account and results in the exponent of the underlying master distribution equalling $\alpha-3$. This result is discussed in more detail in Sec. III B.

With the knowledge of the shape $(1 / \gamma)$ and scale $\left(\sigma_{1}^{*}\right.$ via Eq. (4) for each indenter radius) parameters, the corresponding Weibull distribution is completely defined for each indenter radius. Fig. 1(b) plots the corresponding Weibull CDFs along with the experimental nano-indentation data (of figure 10 in Sudharshan Phani et al. ${ }^{30}$ ) showing a reasonable agreement with the experiment down to an indenter radius of $3.75 \mu \mathrm{m}$. It is emphasized that the shown Weibull cumulative distribution functions are not fitted directly to the data in Fig. 1(b), but rather obtained (via their shape and scale parameters) from a fit of Eq. (6) to the data of Fig. 1(a).

For the larger indenter radii, Morris et al. ${ }^{29}$ consider a plastic model characterized by a density of defects $\left(\rho_{\text {defect }}\right)$ and their mean critical stress. In their work, the statistical size effect with respect to indenter radii is seen to originate from the probability that there exists, within the plastic zone beneath the indenter, at least one defect which has this mean critical stress. If this is the case, then a pop-in will occur with certainty. The random aspect arises from the assumption that the defects are uncorrelated in their spatial position and therefore the probability of encountering one such defect (and therefore a pop-in event) follows a Poisson distribution with a mean $\rho_{\text {defect }} V$, with $V$ being the relevant plastic volume. Thus, the fundamental stochastic construct is, given a well defined critical stress, how likely is it that a volume $V$ is encountered beneath the indenter which induces the pop-in with certainty? The present work considers the reverse construct; given a volume $V$, what critical stress is encountered beneath the indenter that induces the pop-in with certainty? Thus, instead of a Poisson distribution of volumes, a master distribution of critical stresses is assumed. From this perspective, both approaches are equally viable and compatible.

The absence of the predicted scaling and resulting Weibull CDFs for the two smallest indenter radii suggests a failure of the entire extreme-value-statistics approach at these shortest length-scales. In quite general terms, the validity of the current approach is expected to break down at some minimum length scale due to either the dominance of boundary effects of the small plastic volume or the entry of a homogeneous dislocation nucleation regime. In fact, the work of Sudharshan Phani et $a l .^{30}$ has attributed precisely the latter scenario to the two smallest indenter radii considered in the study by Morris et al. ${ }^{29}$
The results of Appendix B are now used to extract an estimate for $\rho$. For the case of the experimental nanoindentation data, Fig. 5 gives $l[\gamma=0.225]=0.136$, giving $\rho=\bar{l} / l$ $\approx 0.12(\mu \mathrm{m})^{-3}=1.1 \times 10^{17}\left(\mathrm{~m}^{3}\right)$ and $\sigma_{0}=\rho^{\gamma} \bar{\sigma}_{0} \approx 16.7 \mathrm{GPa}$. Using $M=\rho r^{3}, M \simeq 39247493,1044616,5339,613,33,6$, 0.4 , and 0.02 for respective indenter radii of 700, 209, 36, $17.5,6.62,3.75,1.5$, and $0.58 \mu \mathrm{m}$. For the larger indenter radii, $M$ becomes quite a large number, and it is justified to employ an extreme value statistics framework to describe the statistics of the pop-in stresses. However, as the indenter radius reduces, $M$ rapidly decreases, eventually to below unity for the two smallest indenter sizes. This latter regime is clearly outside the present formalism based on the statistics of the extreme and, as discussed in the previous paragraphs, a regime of indenter sizes which Sudharshan Phani et al. ${ }^{30}$ have attributed to a change in the underlying microscopic deformation mechanism. Such a change in mechanism with decreasing indenter size is well known. ${ }^{37}$ The estimated density of available plastic events, $\rho$, is one order of magnitude larger than that in Morris et al. ${ }^{29}$-an expected discrepancy since the plastic volume beneath the indenter can be between one and two orders of magnitude larger than $r^{3}$. Alternatively, interpreting $\rho$ directly in terms of indenter volume, the length scale $(1 / \rho)^{1 / 3} \simeq 2 \mu \mathrm{m}$ is precisely the indenter radius below which a change of mechanism has been proposed and for which the present analysis does not work. Because of the rapid increase in $M$ with indenter radius, no improvement on the predicted critical stress statistics could be gained by using the exact expression for $P_{M}[\sigma]$ (Eq. (C5)).

\section{B. The first critical stress in a dislocation dynamics simulation}

The dislocation dynamics simulation method offers one way to model the structural evolution at the resolution of dislocations. ${ }^{38-41}$ Such models, in up to three spatial dimensions, take into account the far-field elastic interaction between dislocations and in many cases also the near-field dislocation interactions such as annihilation, nucleation, and more general reactions leading to dislocation multiplication.

The dislocation model used presently considers a single plane of $N$ infinitely straight edge dislocations under periodic boundary conditions, with periodicity $d$. In addition to their mutual elastic interaction, each dislocation experiences a static sinusoidal stress field characterized by a stress amplitude $\tau_{0}$ and wavelength $\lambda_{0}$. Such an internal field can be viewed as a static mean-field representation of the immobile dislocation content, and the $N$ dislocations as the dynamic mobile dislocation content. Prior to loading, the explicit mobile dislocation configuration is created by randomly placing the $N$ dislocations within the system length and relaxing to a local minimum energy configuration. Although simple, such a model is able to capture a number of features of more complex two and three dimension dislocation dynamics simulations, such as a well defined micro-plastic regime that exhibits an avalanche behaviour, and a transition to a plastic flow regime. For more details, see Ref. 5 which 
investigated a dipolar-mat geometry rather than the single slip plane system mainly considered here.

For the present work, a deformation curve is obtained via the application of a constant stress rate. During loading, the dislocation configuration evolves according to an overdamped dynamics characterized by a friction coefficient whose inverse is the system's mobility parameter. The parameters presently used are those of $\mathrm{Cu}$ and the same used as in the work of Derlet and Maaß. ${ }^{5}$

Fig. 2(a) displays the representative stress strain curves for a number of different system sizes. For all deformation curves, $\tau_{0}, \lambda_{0}$, and the number of mobile dislocations per unit $\lambda_{0}$ are the same. In particular, $\tau_{0}=10 \mathrm{MPa}, \lambda_{0}=2 \mu \mathrm{m}$, and the number of mobile dislocations per unit $\lambda_{0}$ is equal to one. The figure demonstrates that with decreasing system size, $d$, the stress strain curves become increasingly intermittent and more stochastic. The stress-strain curves for the larger system sizes converge to a loading response with a yield stress of approximately half that of $\tau_{0}$. Derlet and Maa $\beta^{5}$ have shown that this yield stress, and at what total strain it occurs, is a function of $\tau_{0}, \lambda_{0}$, and dislocation density.

To investigate the viability of the developed formalism for this model system, the first critical stress is measured for
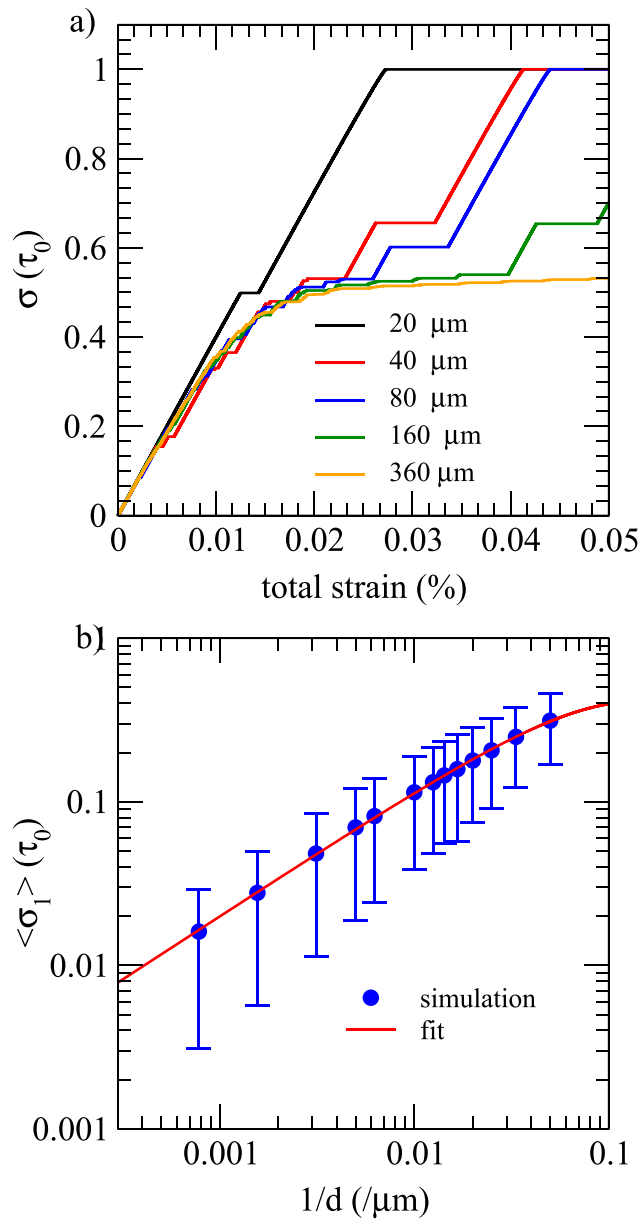

FIG. 2. (a) Stress versus total strain behaviour derived from dislocation dynamics simulations for a range of periodicity lengths at fixed mobile dislocation density. (b) Average first critical stress as a function of the inverse periodicity length derived from 2000 loading curves for each system length and the overall fit (show in red) to Eq. (6). a range of system sizes spanning $d=20 \mu \mathrm{m}$ (10 mobile dislocations) to $d=1280 \mu \mathrm{m}$ (640 mobile dislocations) at a constant dislocation density using the same parameters as in Fig. 2(a). For each $d$, two thousand loading sequences up to the first plastic event are simulated resulting in two thousand critical stress values. Fig. 2(b) displays the average critical stress sequence plotted as a function of $1 / d$ as a log-log plot. The error bars correspond to the associated variances and reflect the increased stochasticity with decreasing system size. Also shown in the figure is an optimal fit of Eq. (6) which, as for the case of the nanoindentation data, describes the observed scaling very well. The optimal parameters are $\gamma=0.775 \pm 0.008, \bar{l}=5.852 \pm 0.606 \mu \mathrm{m}$, and $\bar{\sigma}_{0}=42.362$ $\pm 2.021 \mathrm{MPa}(\mu \mathrm{m})^{-\gamma}$.

The predicted Weibull distributions are plotted (solid curves) in Fig. 3(a) and 3(b) along with histograms (symbols) derived from the 2000 measured critical stresses for each system size. Inspection of Fig. 3(a) demonstrates a good agreement between the theory and simulation for the larger system sizes. However, as the system size decreases, poorer agreement is gradually observed in Fig. 3(b),
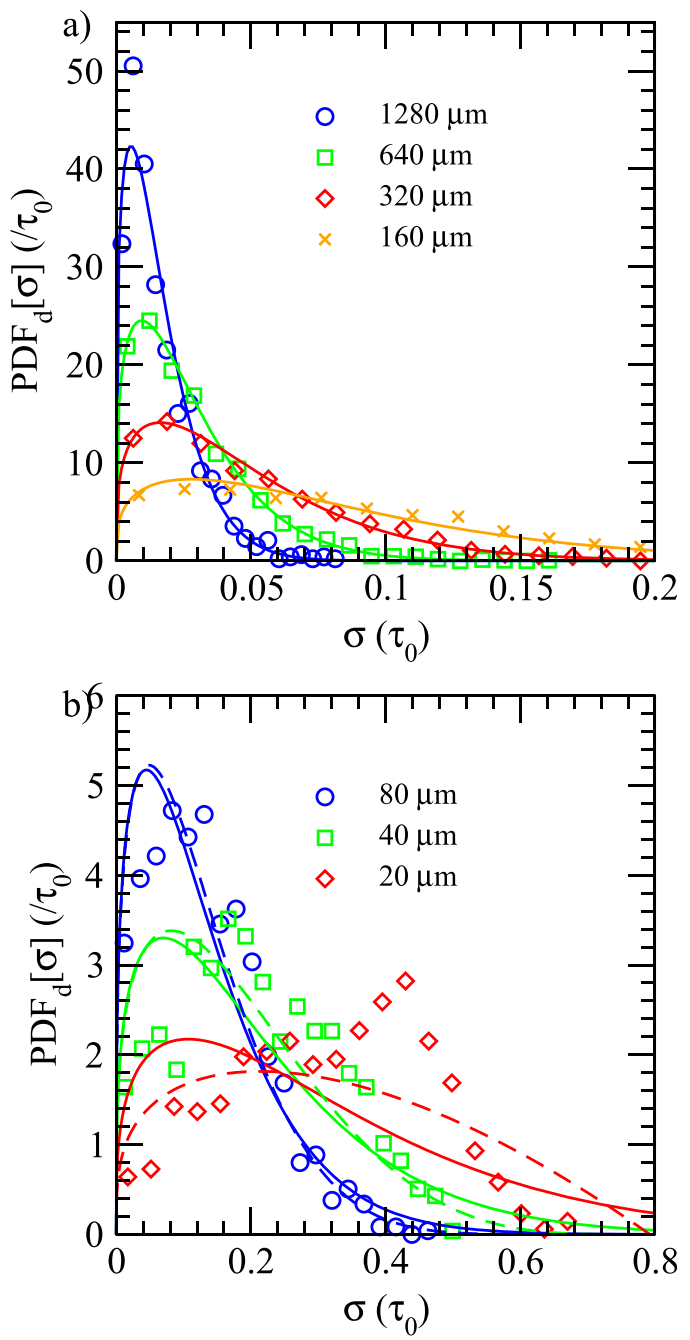

FIG. 3. (a) and (b) Plot of the dislocation dynamics simulation critical stress histogram data for a range of system lengths with corresponding predicted Weibull probability distributions (solid lines of similar colour). In (b), the dashed lines represent the exact probability distribution for the first critical stress (Eq. (A1)). 
particularly for the case when the periodicity length is less than $80 \mu \mathrm{m}$.

The results of Appendix B are now used to extract an estimate for $\rho$. For the case of simulation, Fig. 5 gives $l[\gamma=0.775] \approx 0.673$ giving $\rho=\bar{l} / l \approx 0.12(\mu \mathrm{m})^{-1}$ and $\sigma_{0}=\rho^{\gamma} \bar{\sigma}_{0} \approx 7.9 \mathrm{MPa}$. Via $M=\rho d, M \simeq 147,74,36,18,9$, 5 , and 2 for respective system lengths of $d=1280,640,320$, $160,80,40$, and $20 \mu \mathrm{m}$. Thus, for the smaller system lengths, $M$ is comparable to or less than ten, suggesting the asymptotic Weibull result should not work-as is the case in Fig. 3(b) for $d<80 \mu \mathrm{m}$. When $P[\sigma]$ is assumed to be quadratic (Eq. (C1)), the exact extreme-value statistics distribution for a given $M$, Eq. (A1), may be constructed. Fig. 3(b) plots these exact distributions for the values of $M=9,5$, and 2 for respective system lengths of $d=80,40$, and $20 \mu \mathrm{m}$ as dashed lines. With these exact distributions, agreement is improved when compared to the corresponding Weibull distributions, although for the $d=20 \mu \mathrm{m}(M=2)$ case, a significant discrepancy still exists. Since $P_{M \rightarrow 1}[\sigma] \rightarrow P[\sigma]$, in this regime of small $M$, the statistics of the first critical stress will be increasingly dependent on the actual high-stress shape of the master distribution. Appendix B assumes that this distribution is a power-law cut off at $\sigma_{0}$ (here $\approx 0.79 \tau_{0}$ ); however, at higher stresses, the true master distribution is expected to deviate from the assumed power-law distribution and terminate more smoothly. The discrepancy seen in Fig. 3(b) for the $d=20 \mu \mathrm{m}$ therefore suggests a distribution that has a higher probability density than that of a simple power-law and therefore a smooth termination that occurs somewhat below $\sigma_{0}$.

A value of $\rho \approx 0.12$ corresponds to a mean distance between the plastic events equal to approximately $9 \mu \mathrm{m}$. This represents one plastic event per every four $\lambda_{0}$ units. The dislocation density used for the present simulations corresponds to one dislocation per $\lambda_{0}$ unit. Inspection of the explicit dislocation dynamics reveals, for the larger system sizes, the first discrete plastic event is located in regions containing either two or three dislocations within one $\lambda_{0}$ unit. $^{42}$ Given that the dislocation configuration prior to loading is randomly chosen, the average spacing between configurations involving three dislocations within one $\lambda_{0}$ unit is approximately three $\lambda_{0}$ units-a number quite compatible with the obtained estimate of $1 / \rho$.

To investigate the role of a changing $\rho$ as a function of a fixed system length, the single dislocation dynamics simulations are repeated for the dipolar mat geometry (considered in the study by Derlet and Maa $\beta^{5}$ ) which adds a second parallel slip plane to the one dimensional system. This second slip plane has dislocations with a burgers vector whose direction is anti-parallel to those in the first slip plane. Within each slip plane, the dislocation density, and therefore dislocation number, is the same; however, the total dislocation number for a given periodic length now increases by a factor of two. In this geometry, dislocations interact within each slip plane and across the two different slip planes. Following the study by Derlet and Maaß $\beta^{5}$, the distance between the two slip planes is chosen to equal the mean distance between dislocations within the slip planes.
Fig. 4 plots the first critical stress as a function of the inverse periodic length for both the dipolar mat and a single slip plane (already shown in Fig. 2(b)) simulations. Inspection of this figure shows that, with the addition of a second slip plane, the scale of the first critical stresses reduces. Fitting Eq. (6) to these data gives $\gamma=0.818 \pm 0.010$, $\bar{l}=2.834 \pm 0.772 \mu \mathrm{m}$, and $\bar{\sigma}_{0}=31.148 \pm 1.816 \mathrm{MPa}(\mu \mathrm{m})^{-\gamma}$. From $\mathrm{C}, l[\gamma=0.818]=\bar{l} / l \approx 0.727$ giving $\rho \approx 0.26(\mu \mathrm{m})^{-1}$ and $\sigma_{0}=\rho^{\gamma} \bar{\sigma}_{0} \approx 10.2 \mathrm{MPa}=1.02 \tau_{0}$. Hence, with the addition of a second slip plane, the density of the available plastic events increases by a factor of approximately 2.2 whilst the parameters $\gamma$ and $\sigma_{0}$ change by much less. These numbers are not so different to what one would expect when switching off the inter-plane interactions, which would trivially give no change in $\gamma$ and $\sigma_{0}$, and a factor of two change in $\rho$. A doubling of $\rho$ entails a doubling of $M$ for a given system length, and thus a closer proximity to the Weibull limit-a trend reflected in Fig. 4, which shows a reduced exponential truncation for the dipolar mat data.

\section{DISCUSSION AND CONCLUDING REMARKS}

Sec. III finds the exponent of the master distribution is $\alpha-3 \simeq 0.44$ for the case of the experimental nanoindentation data, whilst for the dislocation dynamics simulations, a value of $\alpha \simeq 0.30$ is obtained. If the two exponents were the same, it could be argued that the probed local instability in both cases is associated with the physics of a single slip system. The one dimensional dislocation network considered in Sec. II then models the case of a single effective slip plane in which the applied shear stress is the resolved shear stress on that plane. Here, the internal static stress field used in Sec. II would then characterize the effect of the surrounding three dimensional network. For the complex threedimensional geometry of the nano-indentation experiment, what is actually measured is the indentation depth and load at the first pop-in, from which a maximum resolved shear stress is calculated and taken as the stress at which the popin occurred. This naturally represents a non-trivial average

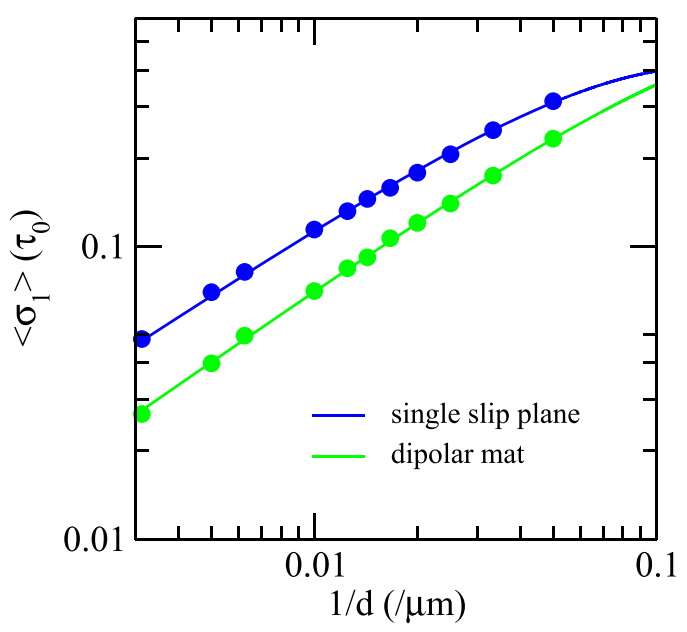

FIG. 4. Average first critical stress as a function of the inverse periodicity length derived from 2000 loading curves for each system length for both the single plane (blue data) and dipolar mat (green data) system. Optimal fits to Eq. (6) are also shown as solid lines. 
over many slip planes. However, this would only affect the pre-factor and not change the exponent of the effective distribution from that of a single slip plane.

The actual differences between the exponents must therefore originate from more fundamental considerations such as the universality class the plasticity belongs to. Indeed, the one-dimensional dislocation model system considered here belongs to a mean field depinning universality class in which the first plastic event is expected to be far from the tuned-criticality occurring at the depinning stress (see Ref. 5). On the other hand, more complex dislocation simulations suggest a non-mean field universality class for which criticality occurs at all stages of deformation. ${ }^{7}$ If the dislocation network is in a state of criticality at the very first plastic event, then marginal stability indicates the value of $\alpha$ is a universal exponent. ${ }^{33}$ To date, no marginal stability analysis has given a prediction for $\alpha$ for the case of a dislocation network. The present work therefore gives initial indications of what the exponent might be.

To investigate the extreme value statistics approach to the onset of plasticity further, two avenues are available. For modelling, two and three dimensional dislocation dynamics simulation could give insight into the dependence of the exponent, $\alpha$, on many active and interacting slip-planes, and on the universality class that the dynamics belongs to. Simulation work of this type has shown that if the simulated system is sufficiently large (greater than 5-10 $\mu \mathrm{m}$ ), then a weakest linkextreme value-scenario is observed, whereas below this length-scale, mechanisms specific to the small system size become operative. ${ }^{43,44}$ On the experimental side, a tensile geometry does not suffer from the inherent instabilities of a compression experiment and has a simple stress geometry when compared to the nano-indentation. Therefore, one promising avenue to obtain the statistics of the first critical stress event as a function of system size is through the tensile deformation of metallic columns. Such micro-wires can be prepared via directional solidification and can result in single crystal columns with diameters ranging between $\sim 1$ and $\sim 100 \mu \mathrm{m}$. ${ }^{45-48}$

The current work has demonstrated, for both experimental nano-indentation data and a dislocation dynamics simulation, that the stress scale of the first discrete plastic event follows an inverse power-law of the plastically deforming volume. It is found that the corresponding statistics of the first critical stress event is described by extreme-value-statistics, allowing for the successful prediction of the Weibull statistics using only this scaling relation and the assumption of an underling power-law master distribution of critical stresses. Remarkably, the extracted master-distribution exponent derived from experiment is comparable to that found for the dislocation simulations, which model a single slip system in the presence of a static internal stress field. For smaller systems, the observed deviations away from the asymptotic Weibull limit are exploited to extract an estimate for the density of plastic events.

\section{ACKNOWLEDGMENTS}

P.M.D. thanks W. A. Curtin, J. P. Molinari, and P. D. Ispánovity for fruitful discussions.

\section{APPENDIX A: DERIVATION OF THE WEIBULL DISTRIBUTION}

The probability of choosing a lowest critical stress $\sigma$ when sampling the probability density function $P[\cdot], M$ times, can be written as

$$
P_{M}[\sigma]=M P[\sigma]\left(1-P_{<}[\sigma]\right)^{M-1}
$$

where the factor $\left(1-P_{<}[\sigma]\right)$ gives the probability of not sampling a critical stress less than or equal to $\sigma$. This must occur $M-1$ times with one additional sampling of the probability density function yielding the required lowest critical stress. This latter successful sampling can occur anywhere between the first and $M$ th sample, leading to the factor $M$ in the above equation. For large $M$

$$
\left(1-P_{<}[\sigma]\right)^{M-1} \simeq \exp \left[-M P_{<}[\sigma]\right]
$$

giving

$$
P_{M}[\sigma] \simeq M P[\sigma] \exp \left[-M P_{<}[\sigma]\right]
$$

Writing $P_{<}[\sigma]=f\left[\sigma^{\frac{1}{\gamma}}\right]$ and Taylor expanding $f[\cdot]$ around the value $\left(\sigma_{1}^{*}\right)^{\frac{1}{\gamma}}$, gives

$$
P_{<}[\sigma] \approx f\left[\sigma_{1}^{*}\right]+f^{\prime}\left[\sigma_{1}^{*}\right]\left((\sigma)^{\frac{1}{\gamma}}-\left(\sigma_{1}^{*}\right)^{\frac{1}{\gamma}}\right)
$$

Here, $f^{\prime}[\cdot]$, is the derivative with respect to the argument of $f[\cdot]$ and not $\sigma$. From Eq. (A4), the probability density function in the vicinity of $\sigma_{1}^{*}$ is then approximated by

$$
P[\sigma] \approx \frac{1}{\gamma} f^{\prime}\left[\sigma_{1}^{*}\right](\sigma)^{\frac{1}{\gamma}-1}
$$

With the above approximations, and since $f\left[\left(\sigma_{1}^{*}\right)^{\frac{1}{\gamma}}\right]$ $=P_{<}\left[\sigma_{1}^{*}\right]=1 / M$, Eq. (A3) becomes

$$
P_{M}[\sigma] \simeq \frac{1}{\gamma \sigma_{\mathrm{W}}}\left(\frac{\sigma}{\sigma_{\mathrm{W}}}\right)^{\frac{1}{\gamma}-1} e^{-\left(\sigma / \sigma_{\mathrm{W}}\right)^{\frac{1}{\gamma}}} \exp \left[\left(\frac{\sigma_{1}^{*}}{\sigma_{\mathrm{W}}}\right)^{\frac{1}{\gamma}}-1\right],
$$

where

$$
\sigma_{\mathrm{W}}=\left(\frac{1}{M f^{\prime}\left[\left(\sigma_{1}^{*}\right)^{\frac{1}{\gamma}}\right]}\right)^{\gamma}=\left(\frac{f\left[\left(\sigma_{1}^{*}\right)^{\frac{1}{\gamma}}\right]}{f^{\prime}\left[\left(\sigma_{1}^{*}\right)^{\frac{1}{\gamma}}\right]}\right)^{\gamma} .
$$

For a sufficiently large $M, \sigma_{1}^{*}$ will be small enough to probe the power-law part of the $P[\sigma]$ and Eq. (A7) reduces to $\sigma_{\mathrm{W}}=\sigma_{1}^{*}$. Thus, Eq. (A6) reduces to the Weibull distribution

$$
P_{M}[\sigma]=\frac{1}{\gamma \sigma_{\mathrm{W}}}\left(\frac{\sigma}{\sigma_{\mathrm{W}}}\right)^{\frac{1}{\gamma}-1} e^{-\left(\sigma / \sigma_{\mathrm{W}}\right)^{\frac{1}{\gamma}}}
$$

with $1 / \gamma$ being the Weibull shape parameter and $\sigma_{1}^{*}$ being the Weibull scale parameter, $\sigma_{\mathrm{W}}$. 


\section{APPENDIX B: INTERPRETATION OF VOLUME EXPONENT FOR AN INDENTATION GEOMETRY}

An infinitesimal material volume $\mathrm{d}^{3} \mathbf{r}$ located at $\mathbf{r}$ with a stress equal to $\sigma[\mathbf{r}]$ is first considered. The probability that this region admits a plastic instability is given by $\rho \mathrm{d}^{3} \mathbf{r} P_{<}[\sigma[\mathbf{r}]]$. The criterion for an instability to occur within the material volume $V$ is then given by

$$
\rho \int_{V} \mathrm{~d}^{3} \mathbf{r} P_{<}[\sigma[\mathbf{r}]]=1 .
$$

For a constant stress field, $\sigma_{1}^{*}=\sigma[\mathbf{r}]$, the above equation becomes $\rho V P_{<}\left[\sigma_{1}^{*}\right]=1$ and reduces to Eq. (1).

For an indenter geometry, the internal stress field will have the form $\sigma[\mathbf{r}]=\sigma_{0} f[\mathbf{r} / a]$ (see Ref. 36) where $a$ is the contact radius, and $\sigma_{0}$ is the maximum resolved shear stress under the indenter (see Ref. 29). $\sigma_{0}$ has the scaling $\sim a / r$ where $r$ is the indenter radius. Changing the integration variable to $\overline{\mathbf{r}}=a \mathbf{r}$ and assuming $P_{<}[\sigma] \sim \sigma^{\alpha_{\text {true }}+1}$, the left hand side of Eq. (B1) scales as

$$
\sim \rho a^{3} \sigma_{0}^{\alpha_{\text {true }}+1} \int_{V} \mathrm{~d}^{3} \overline{\mathbf{r}}(f[\overline{\mathbf{r}}])^{\alpha_{\text {true }}+1} \sim \rho r^{3} \sigma_{0}^{\alpha_{\text {true }}+4} .
$$

In the first scaling, the integral is independent of the contact radius $a$ and indenter radius $r$, and only contributes a constant pre-factor. In the second scaling, which ignores the constant integral, $\sigma_{0} \sim a / r$ has been used to express the factor $a^{3}$ in terms of $\sigma_{0}$ and $r$.

Equating Eq. (B2) (via an appropriate constant pre-factor) to unity, Eq. (1) is again obtained but with the effective exponent, $\alpha=3+\alpha_{\text {true }}$, giving

$$
\sigma_{0}^{*} \sim\left(\frac{1}{r^{3}}\right)^{\frac{1}{\alpha+1}}=\left(\frac{1}{r^{3}}\right)^{\gamma} .
$$

Thus, for the case of indentation, the obtained exponent $\alpha$ (from which the shape parameter of the corresponding Weibull distribution is obtained) gives the underlying probability distribution of the material's critical stress as $\alpha_{\text {true }}=\alpha-3$.

\section{APPENDIX C: A POWER-LAW MASTER DISTRIBUTION}

Here, it is assumed that the underlying master distribution is a pure power-law up to a cut-off critical stress $\sigma_{0}$, beyond which it is zero

$$
P[\sigma]=\frac{1}{\gamma \sigma_{0}^{1 / \gamma}} \sigma^{\frac{1}{\gamma}-1},
$$

for $\sigma<\sigma_{0}$. This gives

$$
P_{<}[\sigma]=\left(\frac{\sigma}{\sigma_{0}}\right)^{\frac{1}{\gamma}}
$$

and, via Eq. (1)

$$
\sigma_{1}^{*}=\sigma_{0}\left(\frac{1}{M}\right)^{\gamma}
$$

Eqs. (C1) and (C2), and Eq. (A1), can now be used to construct the exact first critical stress distribution

$$
P_{M}[\sigma]=\frac{M}{\gamma \sigma_{0}^{1 / \gamma}} \sigma^{\frac{1}{\gamma}-1}\left(1-\left(\frac{\sigma}{\sigma_{0}}\right)^{\frac{1}{\gamma}}\right)^{M-1},
$$

from which an exact expression for the average first critical stress is obtained

$$
\begin{aligned}
\left\langle\sigma_{1}\right\rangle[M] & =M \sigma_{0} \frac{\Gamma[1+\gamma] \Gamma[M]}{\Gamma[M+1+\gamma]} \\
& \doteq \Gamma[1+\gamma] \sigma_{0}\left(\frac{1}{M}\right)^{\gamma} \Pi\left[\gamma, \frac{1}{M}\right] .
\end{aligned}
$$

Here, $\Pi[\gamma, 0]=1$ and $\Gamma[\cdot]$ is the gamma function.

Fig. 5(a) plots $\Pi[\gamma, x]$ and $\exp (-l[\gamma] x)$ for the experimental and simulation values of $\gamma$, using the respective fitted values $l[\gamma=0.225]=0.136$ and $l[\gamma=0.775]=0.673$. Thus, $\Pi[\gamma, 1 / M] \approx \exp (-l[\gamma] / M)$ for $M>10$, and Eq. (C6) may be approximated as
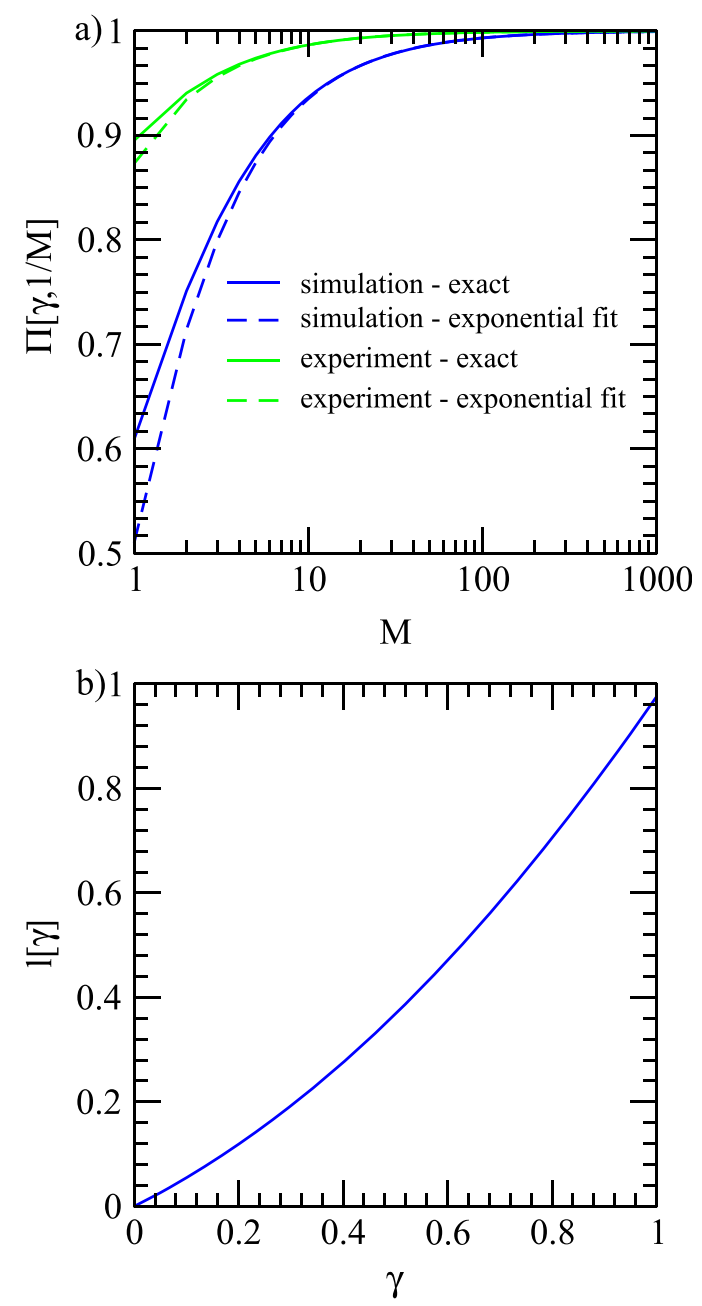

FIG. 5. (a) Solid lines are a plot of $\Pi[\gamma, 1 / M]$ versus $M$ for the simulation exponent $\gamma=0.775$ and the experimental exponent $\gamma=0.225$. The corresponding dashed lines give the approximate representation via $\exp (-l[\gamma] / M)$ with $l[\gamma=0.775]=0.673$ and $l[\gamma=0.225]=0.136$. (b) Plots the numerically determined $l[\gamma]$ versus $\gamma$. 


$$
\left\langle\sigma_{1}\right\rangle[M] \approx \Gamma[1+\gamma] \sigma_{0}\left(\frac{1}{M}\right)^{\gamma} \exp (-l[\gamma] / M),
$$

which is of a similar form to Eq. (5) with the parameter $l$ now depending on $\gamma$. Fig. 5(b) plots the optimal value of $l[\gamma]$ versus $\gamma$ for $0<\gamma \leq 1$.

Using Eq. (C4), Eq. (C7) may be written as

$$
\left\langle\sigma_{1}\right\rangle[M] \approx \Gamma[1+\gamma] \sigma_{1}^{*} \Pi\left[\gamma, \frac{1}{M}\right] .
$$

Inspection of Fig. 5(a) shows that Eqs. (C7) and (C8) tend respectively to Eqs. (3) and (4) for $M>50$, demonstrating the asymptotic Weibull limit remains a good approximation for quite small values of $M$.

${ }^{1}$ J. P. Sethna, K. A. Dahmen, and C. R. Myers, Nature 410, 242 (2001).

${ }^{2}$ M.-C. Miguel, A. Vespignani, S. Zapperi, J. Weiss, and J. R. Grasse, Nature 410, 667 (2001).

${ }^{3}$ J. Weiss and D. Marsan, Science 299, 89 (2003).

${ }^{4}$ F. F. Csikor, C. Motz, D. Weygand, M. Zaiser, and S. Zapperi, Science 318, 251 (2007).

${ }^{5}$ P. M. Derlet and R. Maßß, Simul. Mater. Sci. Eng. 21, 035007 (2013).

${ }^{6}$ P. D. Ispánovity, A. Hegyi, I. Groma, G. Gyorgyi, K. Ratter, and D. Weygand, Acta. Mater. 61, 6234 (2013).

${ }^{7}$ P. D. Ispánovity, L. Laurson, M. Zaiser, I. Groma, S. Zapperi, and M. J. Alava, Phys. Rev. Lett. 112, 235501 (2014).

${ }^{8}$ M. Zaiser, Adv. Phys. 55, 185 (2006).

${ }^{9}$ A. P. Mehta, K. A. Dahmen, and Y. Ben-Zion, Phys. Rev. E 73, 056104 (2006).

${ }^{10}$ K. A. Dahmen, Y. Ben-Zion, and J. T. Uhl, Phys. Rev. Lett. 102, 175501 (2009).

${ }^{11}$ M. D. Uchic, D. M. Dimiduk, J. N. Florando, and W. D. Nix, Science 305, 986 (2004).

${ }^{12}$ D. M. Dimiduk, C. Woodward, R. LeSar, and M. D. Uchic, Science 312, 1188 (2006).

${ }^{13}$ M. D. Uchic, P. A. Shade, and D. M. Dimiduk, Annu. Rev. Mater. Sci. 39, 361 (2009).

${ }^{14}$ N. Friedman, A. T. Jennings, G. Tsekenis, J.-Y. Kim, M. Tao, J. T. Uhl, J. R. Greer, and K. A. Dahmen, Phys. Rev. Lett. 109, 095507 (2012).

${ }^{15}$ R. Maaß, P. M. Derlet, and J. R. Greer, Small 11, 341 (2015).

${ }^{16}$ M. E. Cates, J. P. Wittmer, J.-P. Bouchaud, and P. Claudin, Phys. Rev. Lett. 81, 1841 (1998).

${ }^{17}$ A. J. Liu and S. R. Nagel, Nature 396, 21 (1998).
${ }^{18}$ W. Weibull, J. Appl. Mech. 18, 293 (1951).

${ }^{19}$ W. Weibull, Appl. Mech. Rev. 5, 449 (1952).

${ }^{20}$ E. J. Gumbel, Statistics of Extremes (Columbia University Press, New York, 1958).

${ }^{21}$ R. A. Fisher and L. H. C. Tippett, Proc. Cambridge Philos. Soc. 24, 180 (1928).

${ }^{22}$ B. Gnedenko, Ann. Math. 44, 423 (1943).

${ }^{23}$ B. Chalmers, Proc. R. Soc. London, Ser. A 156, 427 (1936).

${ }^{24}$ R. F. Tinder and J. Washburn, Acta Metall. 12, 129 (1964).

${ }^{25}$ R. F. Tinder and J. P. Trzil, Acta Metall. 21, 975 (1973).

${ }^{26}$ P. M. Derlet and R. Maaß, Philos. Mag. 95, 1829 (2015).

${ }^{27}$ R. Maaß, C. Volkert, and P. M. Derlet, Scr. Mater. 102, 27 (2015).

${ }^{28}$ J. D. Eshelby, Proc. R. Soc. London, Ser. A 241, 376 (1957).

${ }^{29}$ J. R. Morris, H. Bei, G. M. Pharr, and E. P. George, Phys. Rev. Lett. 106, 165502 (2011).

${ }^{30}$ P. Sudharshan Phani, K. E. Johanns, E. P. George, and G. M. Pharr, J. Mater. Res. 28, 2728 (2013).

${ }^{31}$ H. A. David and H. N. Nagaraja, Order Statistics, Wiley Series in Probability and Statistics (Wiley, 2003).

${ }^{32}$ J.-P. Bouchaud and M. Méezard, J. Phys. A: Math. Gen. 30, 7997 (1997).

${ }^{33}$ M. Müller and M. Wyart, Annu. Rev. Condens. Matter Phys. 6, 177 (2015).

${ }^{34}$ K. L. Johnson, Contact Mechanics (Cambridge University Press, Cambridge, England, 1985), p. 92.

${ }^{35}$ C. Anthony and C. Fisher, Introduction to Contact Mechanics (Springer, New York, 2000), p. 89.

${ }^{36}$ H. Bei, Z. P. Lu, and E. P. George, Phys. Rev. Lett. 93, 125504 (2004).

${ }^{37}$ E. T. Lilleodden and W. D. Nix, Acta Mater. 54, 1583 (2006).

${ }^{38}$ E. Van der Giessen and A. Needleman, Modell. Simul. Mater. Sci. Eng. 3, 689 (1995).

${ }^{39}$ D. Weygand, L. H. Friedman, and E. Van der Giessen, Modell. Simul. Mater. Sci. Eng. 10, 437 (2002).

${ }^{40}$ B. Devincre, L. Kubin, and T. Hoc, Scr. Mater. 54, 741 (2006).

${ }^{41}$ V. V. Bulatov and W. Cai, Computer Simulations of Dislocations (Oxford University Press, Oxford, 2006).

${ }^{42}$ P. M. Derlet and R. Maaß, Phys. Rev. E 94, 033001 (2016).

${ }^{43}$ P. S. S. Leung, H. S. Leung, B. Cheng, and A. H. W. Ngan, Modell. Simul. Mater. Sci. Eng. 23, 035001 (2015).

${ }^{44}$ J. A. El-Awady, M. Wen, and N. M. Ghoniem, J. Mech. Phys. Solids. 57, 32 (2009).

${ }^{45}$ H. Bei, R. I. Barabash, G. E. Ice, W. Liu, J. Tischler, and E. P. George, Appl. Phys. Lett. 93, 071904 (2008).

${ }^{46}$ P. Sudharshan Phani, K. E. Johanns, G. Duscher, A. Gali, E. P. George, and G. M. Pharr, Acta Mater. 59, 2172 (2011).

${ }^{47}$ K. E. Johanns, A. Sedlmayr, P. Sudharshan Phani, R. Mönig, O. Kraft, E. P. George, and G. M. Pharr, J. Mater. Res. 27, 508 (2012).

${ }^{48}$ J. Krebs, "Cast aluminium microwires: processing and plastic deformation," Ph.D. thesis 6482 (EPFL, 2015). 